\title{
Estudiantes en contextos de educación a distancia. Variables vinculadas con el logro académico
}

\section{(Students in distance education contexts. Variables linked to academic achievement)}

\author{
Analía Claudia Chiecher Costa \\ Universidad Nacional de Río Cuarto (Argentina)
}

DOI: http://dx.doi.org/10.5944/ried.22.2.23368

\section{Cómo citar este artículo:}

Chiecher Costa, A. (2019). Estudiantes en contextos de educación a distancia. Variables vinculadas con el logro académico. RIED. Revista Iberoamericana de Educación a Distancia, 22(2), pp. 203-223. doi: http://dx.doi.org/10.5944/ ried.22.2.23368

\section{Resumen}

El artículo toma como punto de partida las altas cifras de abandono que caracterizan a la educación a distancia y, por el contrario, los bajos porcentajes de estudiantes que consiguen cursar trayectorias ajustadas a los tiempos que establece el plan de estudios. El objetivo del escrito se orienta a analizar perfiles de un grupo de estudiantes de primer año, de modalidad a distancia, cuyas trayectorias se ciñeron a la progresión temporal fijada por el plan de estudios. Se realizó un estudio no experimental y descriptivo en el cual participaron estudiantes de primer año de carreras de grado dictadas a distancia, quienes cumplían, además, la condición de haber regularizado todas las asignaturas previstas en el plan de estudios. Para la recolección de datos se administró un cuestionario de autoinforme, que recaba información sobre variables personales y contextuales con posible incidencia en las trayectorias académicas. Los resultados muestran que, entre las variables personales consideradas en el estudio, aquellas que parecen tener vinculación con trayectorias de logro son el nivel educativo de los padres, las metas de aprendizaje y las atribuciones causales internas y modificables. Asimismo, las variables contextuales percibidas como promotoras del logro se vincularon principalmente con la disponibilidad del profesor y su rapidez para responder consultas y ofrecer devoluciones de las actividades realizadas. La discusión propone líneas de acción que, sustentadas en los resultados del estudio, podrían contribuir a la promoción del logro estudiantil en la educación a distancia. 
Palabras clave: educación; universidad a distancia; estudiantes universitarios de primer ciclo; alto rendimiento.

\begin{abstract}
The article takes as a starting point the high rates of dropouts that characterize distance education, in contrast with the low percentages of students who can conclude their degree courses on time. The objective of this article is to analyze the profiles of a group of students of the first year in distance education whose studies could follow the fixed time of their career. A non-experimental and descriptive study was carried out in which first-year students of distance degree courses took part. This group of students fulfilled the condition of having regularized all the subjects included in the curriculum. For data collection, a self-report questionnaire was administered that collects information on personal and contextual variables with possible impact on academic trajectories. The results show that the personal variables which have a connection with achievement include the educational level of parents, the learning goals, and the internal and modifiable causal attributions. Likewise, contextual variables which are considered as promoters of achievement are mainly related to the availability of the professor and the speed to answer queries and offer feedback to the activities proposed. Lines of action are proposed that, based on the results of the study, are expected to contribute to the promotion of student achievement in distance education.
\end{abstract}

Keywords: education; open university; undergraduate students; high achievement.

Las tecnologías de la información y la comunicación nos rodean, nos envuelven, son parte de los escenarios donde cotidianamente nos movemos $\mathrm{y}$, si bien suele asociarse el uso de tecnologías con los jóvenes, lo cierto es que ya no existe generación que no las utilice.

Ahora bien, ¿cualquier persona habituada a usar tecnologías puede tornarse un estudiante exitoso en un contexto de educación a distancia? o ¿̇son necesarias otras habilidades, características y competencias -más allá de las tecnológicas- para desempeñarse y aprender exitosamente en un entorno virtual? Más aún, ¿̇el éxito de un estudiante virtual depende solamente de las habilidades que él mismo pueda aportar? o ¿̇puede promoverse el logro académico desde el contexto?

A juzgar por datos concretos acerca de las tasas de abandono en esta modalidad de estudios, así como por resultados de investigaciones previas, estudiantes con determinados perfiles sociodemográficos, cognitivos, motivacionales, y con ciertas habilidades tecnológicas, tendrían mayores oportunidades de cursar exitosamente y finalizar estudios a distancia. Del mismo modo, ciertas variables del contexto, de orden institucional e instruccional, también podrían operar en favor del logro académico. Presentamos en los próximos apartados algunos datos que apoyan esta premisa. 


\section{MUCHOS NO LLEGAN A LA META}

El abandono de los estudios es un problema que afecta de manera generalizada al nivel de educación superior. No obstante, en la modalidad a distancia, las cifras resultan más alarmantes aún (Carballo Aguilar, 2017). En tal sentido, Rivera (2011) presenta algunas tasas internacionales de deserción en la educación a distancia, mostrando que, en líneas generales, las instituciones universitarias que ofrecen cursos a distancia suelen perder la mitad, o incluso más, de los estudiantes matriculados.

En nuestro contexto local -la Universidad Nacional de Río Cuarto, en Argentinalas cifras no escapan a la tendencia señalada. De hecho, se dictan carreras del área de Ciencias Económicas en modalidad a distancia y, como en otras instituciones que ofertan formación en esta modalidad, preocupan altamente las cifras vinculadas con el abandono de los estudios. Así, aproximadamente el 70\% de los estudiantes que inician tales carreras no se inscribe en el siguiente ciclo lectivo en asignaturas del segundo año, lo cual hace suponer que se trata de 'desertores' o de sujetos con alto riesgo de abandono (Ficco, Chiecher y Bersía, 2013).

Si miramos desde una óptica diferente y nos concentramos en los estudiantes de modalidad a distancia que logran cursar exitosamente el primer año de estudios, las cifras son también contundentes. Solamente entre un $8 \%$ y $10 \%$ de los inscriptos entre 2015 y 2017 consiguieron regularizar las asignaturas correspondientes al primer cuatrimestre. Comparativamente, para el mismo periodo, en la modalidad presencial de las mismas carreras, los porcentajes de estudiantes que lograron regularizar todas las asignaturas del primer cuatrimestre oscilan entre $36 \%$ y $43 \%$. Evidentemente, estudiar a distancia no resulta sencillo.

Ahora bien ¿cómo sostienen sus trayectorias de logro aquellos estudiantes que -aunque pocos en cantidad- avanzan en el cursado de las carreras a distancia al ritmo previsto por el plan de estudios? ¿Qué rasgos los definen? ¿Qué dicen sus voces acerca de cuáles son los factores o ayudas externas que contribuyen al éxito?

Dada la escasa cantidad de sujetos que consiguen trayectorias de logro en la modalidad a distancia parece promisorio conocer las variables que contribuyen a sostenerlo. Así, para atender a la prevención del abandono y el fortalecimiento de la permanencia, resulta valioso hacer foco en las trayectorias de alumnos con buenos resultados académicos y en las variables que contribuyen a explicarlo. Un conocimiento profundo y contextualizado de las trayectorias exitosas, conformará el punto de partida a partir del cual pensar en acciones para fortalecer la permanencia y prevenir el abandono.

En el contexto descripto, este artículo presenta avances en el conocimiento de algunos de los rasgos típicos que caracterizan a los estudiantes con trayectorias de logro en el primer año universitario y, específicamente, en la modalidad de educación a distancia. Asimismo, se consideran las percepciones de estos sujetos acerca de aquellos factores del contexto que contribuyen y apoyan el éxito académico. 


\section{UNOS POCOS CONSIGUEN ALCANZAR LA META}

Estudiar a distancia - y hacerlo con éxito- requiere de parte del alumno el despliegue de un conjunto de habilidades y competencias que van más allá de las habilidades tecnológicas para moverse con soltura en un ambiente virtual. De hecho, el estudiante a distancia necesita desplegar habilidades que le permitan comunicarse efectiva y asertivamente, colaborar, expresarse y, en definitiva, aprender.

Las investigaciones previas sobre el tema están más bien enfocadas en identificar variables que operan como causas de deserción (Carballo Aguilar, 2017). Son más escasos los estudios que se focalizan en identificar variables relacionadas con el éxito académico en entornos de educación a distancia. Entre las que están disponibles, destacan como favorecedoras de un alto rendimiento ciertas variables propias de la modalidad, variables vinculadas con la interacción profesor-alumno y características personales del estudiante. Atenderemos a continuación a estos aspectos.

\section{Características de la modalidad favorecedoras del logro académico}

Entre las características propias de la modalidad, capaces de favorecer el logro de los estudiantes, se destaca la flexibilidad temporal y la posibilidad que ofrece la educación a distancia de compatibilizar responsabilidades laborales y familiares, pudiendo el alumno estudiar a su propio ritmo y en el momento que le resulta más oportuno (Rivera, 2011). Ello contribuiría al éxito del estudiante virtual, siempre y cuando éste sea capaz de manejar adecuadamente los tiempos y disponga, efectivamente, de tiempo real para dedicar al estudio.

La dimensión temporal atraviesa fuertemente los procesos de educación a distancia. En efecto, si bien resulta atractiva la flexibilidad y autonomía que ofrece la modalidad, es necesario disponer de "tiempo real" para dedicar a las tareas de un curso. Sobre el tema, Romero y Barberá (2013) advierten acerca de la crucial importancia de la regulación del tiempo que pueda hacer el alumno frente a la flexibilidad temporal que ofrece un campus virtual. Muchos estudiantes se inclinan por la modalidad a distancia precisamente porque ésta es la única alternativa compatible con otras obligaciones laborales y familiares. No obstante, debe disponerse de un tiempo real (diario o al menos regular) que el sujeto pueda dedicar al estudio. Si esta condición no se cumple, parece improbable que alguien logre atender exitosamente a las demandas y propuestas académicas de un curso virtual.

\section{La interacción profesor-alumno en la educación a distancia}

En cuanto a las variables vinculadas con la calidad de las tutorías y las interacciones profesor-alumno, se ha destacado la importancia de una fuerte presencia docente (Chiecher y Donolo, 2013) o presencia didáctica (Garrison, Anderson y Archer, 2000 
y 2003). La presencia docente se vincula fuertemente con la rapidez de las respuestas y retroalimentación que el tutor ofrece a las dudas e inquietudes de los estudiantes. Así, respuestas rápidas y atención permanente e inmediata constituyen un factor determinante para motivar y promover la permanencia en contextos de educación a distancia (Carballo Aguilar, 2017).

Ayudar al aprendizaje virtual no es simplemente presentar información o plantear tareas a realizar por parte del alumno. Es, esencialmente, seguir de manera continuada el proceso de aprendizaje que este desarrolla; monitorear los avances; detectar dificultades o rumbos equívocos; $\mathrm{y}$ ofrecer los apoyos y soportes necesarios en el momento oportuno (Onrubia, 2016). Así, factores como el tiempo que transcurre para que el profesor brinde retroalimentación al estudiante, lo apropiada que sea esa retroalimentación, y la cantidad de comunicaciones relacionadas con el curso, pueden impactar positiva o negativamente en la decisión de persistir o abandonar el curso en línea (Rivera, 2011).

\section{Características personales de los estudiantes que favorecen el logro}

Algunos estudios vinculan el alto rendimiento del estudiante a distancia con variables personales como la motivación intrínseca o hacia el aprendizaje (Benett y Monds, 2008; Chiecher, Ficco, Paoloni y García, 2016; Chiecher, Paoloni y Ficco, 2014), la valoración de las tareas, la autorregulación del aprendizaje (Chiecher, Paoloni y Ficco, 2014; Kizilcec, Pérez y Maldonado, 2017) y la atribución de relevancia a los estudios realizados (Park y Choy, 2009).

Centrándonos en la motivación - aspecto que vamos a considerar en el presente artículo-, investigaciones previas han mostrado que la orientación hacia metas de aprendizaje suele presentarse asociada con el logro académico (Castejón, Gilar, Miñano y Veas, 2016; Chiecher, Ficco, Paoloni y García, 2016; Garbanzo Vargas, 2007; Huertas, 1997; Huertas y Agudo, 2003; Pintrich, 2000). De hecho, estudiantes para quienes el estudio significa más que una tarea un disfrute por el saber suelen orientar sus esfuerzos y comportamientos de manera tal que logran un rendimiento exitoso. En la misma línea, las atribuciones causales que realizan los estudiantes - esto es, las explicaciones que dan, una vez finalizada la actuación, acerca de los resultados obtenidos-, también han sido vinculadas con el rendimiento académico. Así, investigaciones previas han mostrado que asumir que los resultados académicos se deben a causas internas, modificables y controlables (como por ejemplo el esfuerzo y dedicación al estudio), influye en el logro de buenos resultados académicos (Huertas, 1997).

En síntesis, para estudiar a distancia -y para ampliar las posibilidades de llegar a la meta de concluir los estudios- se necesita algo más que saber navegar por Internet. Es necesario manejar el campus virtual que da soporte a la propuesta educativa, conocer los distintos espacios, materiales, foros y recursos, así como su uso correcto y conocer los canales de ayuda que existen. Resultan además competencias 
importantes para el aprendizaje en línea, la de comunicarse efectivamente con docentes y compañeros en los diferentes espacios de interacción y atender a una cierta regularidad en la conexión y lectura de mensajes (Sepúlveda, 2016). También sería conveniente que el alumno que se inscribe para estudiar en modalidad virtual experimente una fuerte motivación por aprender y muestre habilidad para operar con un repertorio de estrategias cognitivas, metacognitivas y de regulación de recursos, que son las que le ayudarán a sostener una trayectoria exitosa (Chiecher, Paoloni y Ficco, 2014).

\section{PROPÓSITOS}

En este artículo interesará particularmente analizar rasgos y perfiles típicos de un grupo de estudiantes de primer año, en modalidad a distancia, cuyas trayectorias fueron exitosas o, en otros términos, se ciñeron a la progresión temporal fijada por el plan de estudios. Nos focalizaremos así en algunas variables sociodemográficas que caracterizan al grupo, así como en variables relativas a aspectos motivacionales. También atenderemos, desdela perspectiva de los sujetos exitosos, a las características que deberían reunir los docentes a cargo de la enseñanza y las tareas que proponen.

\section{METODOLOGÍA}

El estudio - de carácter no experimental y descriptivo- se orientó a identificar características y percepciones del contexto de alumnos de primer año (cohortes 2015, 2016 y 2017) con trayectorias de logro, inscriptos en carreras de Ciencias Económicas dictadas en modalidad a distancia en una universidad pública argentina.

Con el fin de identificar al grupo de estudiantes con trayectorias de logro, se accedió inicialmente a los listados de inscriptos y se realizó un seguimiento de sus trayectorias académicas una vez transcurrido el primer cuatrimestre de cursada. Dicho seguimiento permitió identificar a los estudiantes que habían cursado exitosamente, definidos como aquellos que consiguieron regularizar todas las asignaturas del primer cuatrimestre. Solamente 61 sujetos de un total de 693 que se inscribieron para cursar entre 2015 y 2017 lograron regularizar todas las asignaturas previstas para el primer cuatrimestre; esto es, tan solo un $9 \%$ del total.

\section{Sujetos}

Participaron del estudio 46 estudiantes inscriptos para cursar carreras del área de Ciencias Económicas en modalidad a distancia en una Universidad Argentina. Todos ellos lograron regularizar las asignaturas del primer cuatrimestre de la carrera elegida (son estudiantes con trayectorias de logro) y además dieron respuesta al cuestionario administrado en el marco de este estudio. Cabe destacar que representan 
un 75\% del grupo total de estudiantes con trayectorias de logro de las cohortes 2015, 2016 y 2017 (N=61).

La edad promedio del grupo es de 27,6 años. En la tabla 1 se presenta la distribución por edades. En cuanto al género, 38 son mujeres y 8 son varones.

Tabla 1. Distribución de sujetos con trayectorias de logro según edad

\begin{tabular}{|l|l|}
\hline \multicolumn{1}{|c|}{ Edad } & \multicolumn{1}{c|}{ Frecuencia } \\
\hline 20 años o menos & 8 sujetos \\
\hline Entre 21 y 25 años & 11 sujetos \\
\hline Entre 26 y 30 años & 10 sujetos \\
\hline Entre 31 y 35 años & 13 sujetos \\
\hline 36 años o más & 4 sujetos \\
\hline
\end{tabular}

\section{Instrumento}

Para la recolección de los datos se administró el 'Cuestionario sobre trayectorias exitosas en el primer año universitario', el cual fue elaborado y probado en el marco de un estudio piloto por nuestro equipo ${ }^{1}$. Fue asimismo sometido a evaluación de seis jueces expertos en el tema quienes, en términos generales, acordaron en avalar la pertinencia de los ítems del cuestionario para medir los aspectos que dicen medir. En Bossolasco, Chiecher y Dos Santos (en prensa) puede accederse a mayores detalles vinculados con la validación del instrumento y sus resultados.

El cuestionario se diseñó con un formato de autoadministración, utilizando un formulario online, al cual se accede desde una URL. Incluye diversos ítems, algunos de respuesta libre y abierta, y otros con alternativas de respuesta entre las que el sujeto debe escoger. Inicia con una introducción en la cual se informa sobre sus objetivos, resaltando el interés de la investigación y los beneficios directos o indirectos resultantes de su contestación. Seguidamente se presentan las instrucciones para completarlo y, con el propósito de atender a cuestiones éticas, se informa a los encuestados de que los datos recogidos serán usados guardando la confidencialidad y el anonimato en el marco de investigaciones científicas, excepto si manifestaran expresamente una negativa a ello.

Estructurado en 10 secciones, el cuestionario procura relevar información acerca de diferentes variables - personales y contextuales- a fin de analizar el modo en que se entrelazan en sujetos con trayectorias académicas exitosas. Para los fines de este escrito se tomarán en consideración los datos referidos a algunas variables sociodemográficas que caracterizan al grupo de estudio, aspectos referidos a la motivación y aspectos relativos a las percepciones de los estudiantes acerca de rasgos de los docentes y de las tareas potencialmente favorecedores del logro. 
Se realizarán análisis cuantitativos o cualitativos conforme al tipo de datos recogidos mediante cada uno de los ítems considerados. En el caso de los ítems de respuestas prefijadas por alternativas, se realizarán análisis de frecuencias y porcentajes. En cambio, para el análisis de las respuestas dadas a ítems abiertos, se procederá a la construcción de categorías en base a las recurrencias de las respuestas.

\section{RESULTADOS}

Se presentan en este apartado los principales resultados obtenidos. En primer término, se describe al grupo de estudiantes desde el punto de vista de algunos aspectos personales. En segundo término, se atiende a algunas variables contextuales que los estudiantes con trayectorias de logro perciben como facilitadoras del éxito: entre ellas, las características de los docentes y de las tareas que proponen.

\section{Variables personales}

a. Estado civil. Entre los 46 sujetos encuestados, un 59\% ( $\mathrm{N}=27)$ eran solteros, $32 \%$ estaban casados o unidos de hecho $(\mathrm{N}=15), 7 \%$ divorciados o separados $(\mathrm{N}=3)$ y 1 caso no respondió (Figura 1).

Figura 1. Distribución de estudiantes con trayectorias de logro según estado civil $(\mathrm{N}=46)$

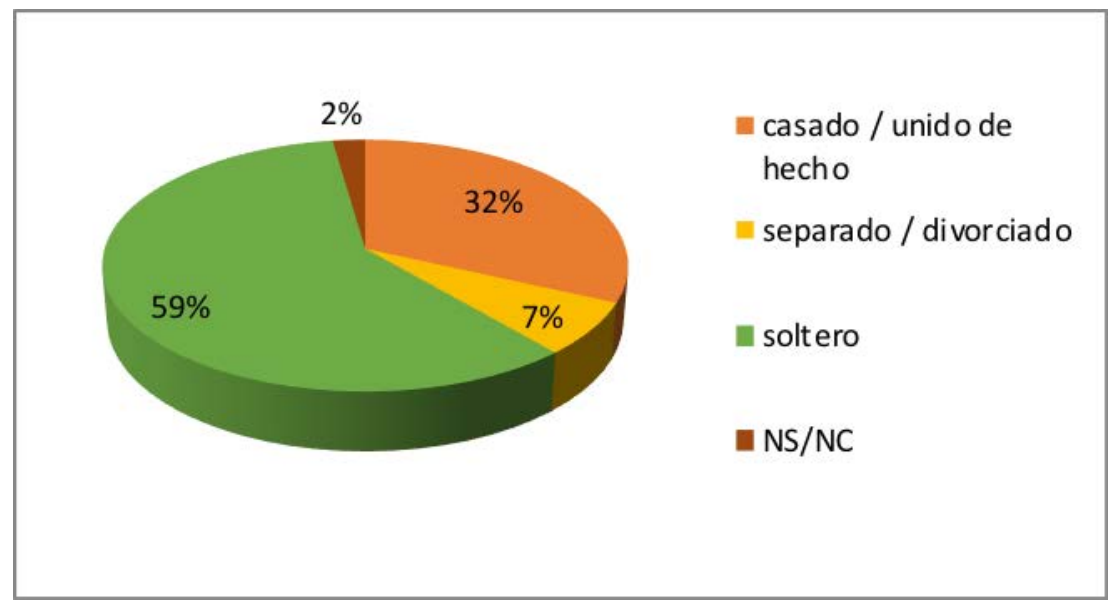

La distribución según estado civil de los sujetos con trayectorias de logro coincide, en términos generales, con el perfil del grupo general de estudiantes que año tras año se inscriben en la modalidad a distancia. En efecto, la tendencia marca 
que mayoritariamente los estudiantes son solteros. No sería ésta, entonces, una variable altamente relacionada con las trayectorias académicas de los estudiantes.

b. Cantidad de hijos. Más de la mitad de los 46 estudiantes con trayectorias de logro no tenían hijos ( $52 \%$; $\mathrm{N}=24$ ), en tanto que el $48 \%$ restante tenía entre 1 y 4 hijos (Figura 2).

Figura 2. Distribución de estudiantes con trayectorias de logro según cantidad de hijos

$(\mathrm{N}=46)$

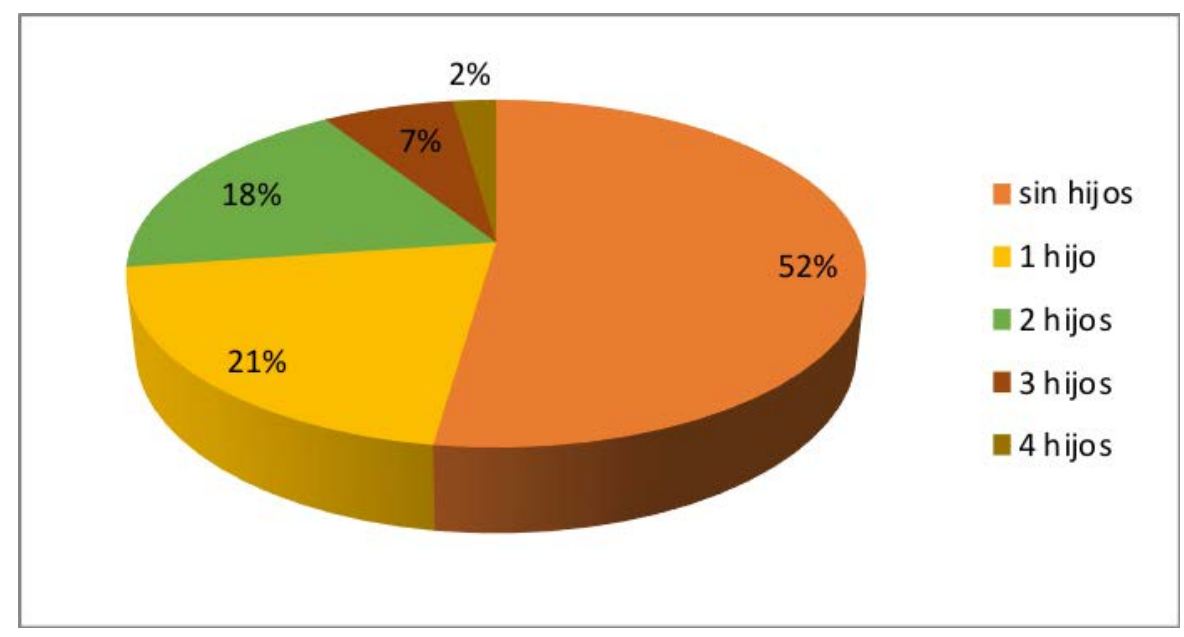

También en este aspecto la distribución de este grupo de estudiantes con trayectorias de logro se parece a la del grupo general de estudiantes de nuevo ingreso. No estaríamos frente a una variable con alto impacto sobre las trayectorias de los estudiantes.

c. Orientación del secundario. Un $43 \%$ del grupo $(\mathrm{N}=20)$ cursó estudios secundarios en escuelas con orientación afín a la carrera elegida (economía, administración, contabilidad, gestión). El resto se distribuyó en otras orientaciones con menor afinidad en relación con las ciencias económicas (Figura 3). 
Figura 3. Distribución de estudiantes con trayectorias de logro según orientación secundario $(\mathrm{N}=46)$

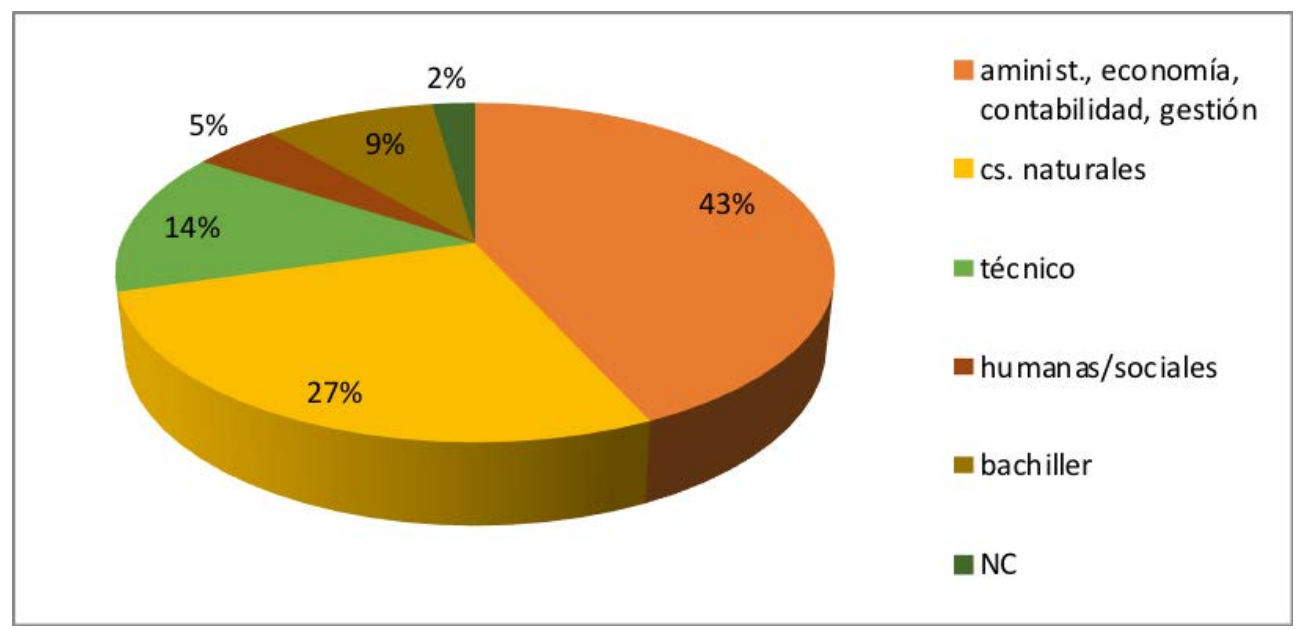

También este aspecto se presenta de modo similar en el grupo general de estudiantes. Por tal motivo, podría decirse que la orientación del secundario cursado por los estudiantes no estaría asociada de manera directa con trayectorias más o menos exitosas.

d. Nivel educativo de los padres. Un grupo mayoritario $(39 \%, \mathrm{~N}=18)$ tienen madres y padres que han completado el primario o han avanzado en el nivel secundario, aunque sin finalizarlo. Por debajo de esta cifra, 16 padres (35\%) y 15 madres (33\%) de estos chicos con trayectorias de logro han completado el secundario o bien iniciado estudios universitarios (Figura 4). 
Figura 4. Distribución de estudiantes con trayectorias de logro según nivel educativo de los padres $(\mathrm{N}=46)$

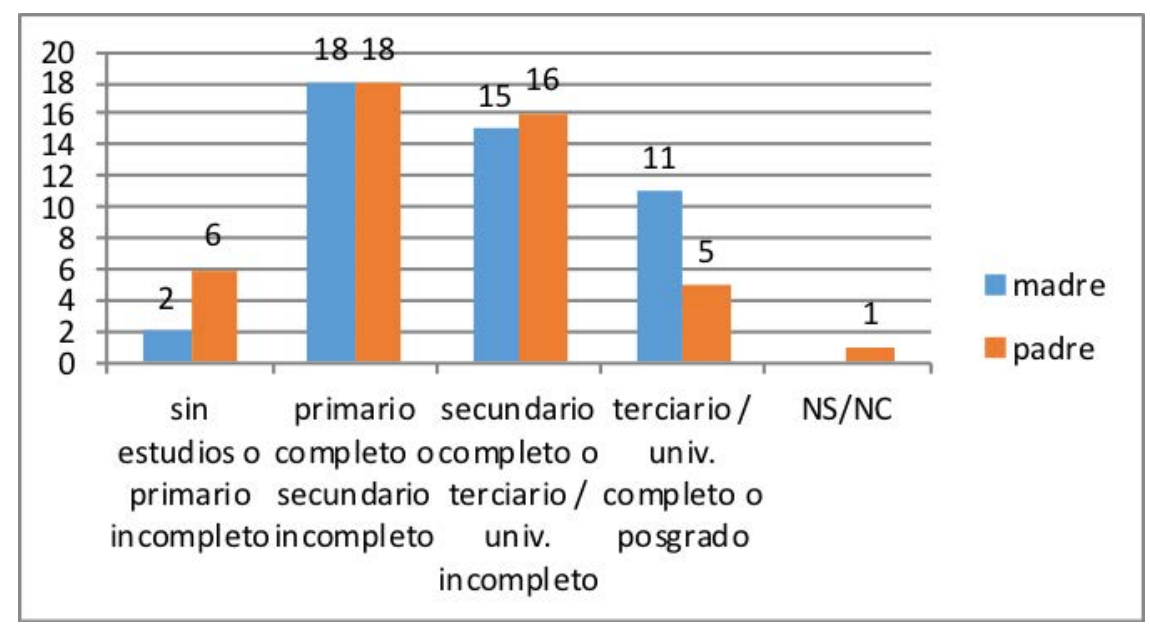

Estos datos están en la línea de los obtenidos respecto del grupo general de estudiantes. Sin embargo, un dato llamativo es que la cantidad de madres y padres con estudios superiores se duplica en el grupo que estamos estudiando en comparación con el grupo de estudiantes en general; así, en el grupo de estudiantes con trayectorias de logro el $24 \%$ de las madres y el $\mathbf{1 1} \%$ de los padres tienen título de nivel superior, mientras que en el grupo general los porcentajes rondan la mitad tanto para padres como para madres.

e. Trabajo durante los estudios. En el momento de ser encuestados, esto es, una vez finalizado el primer cuatrimestre de cursado, un $67 \%(\mathrm{~N}=31)$ trabajaba en tanto que el restante $33 \%(\mathrm{~N}=15)$ no lo hacía. Entre quienes trabajaban, la mayor parte lo hacía en relación de dependencia (81\%), desempeñándose mayormente en actividades vinculadas con la carrera que cursaban (77\%). Llamativamente un alto porcentaje declaró compatibilizar el estudio con actividades laborales demandantes en tiempo; como puede observarse, el mayor porcentaje del grupo (35\%) informó una dedicación semanal al trabajo que va de 31 a 40 horas e incluso un $33 \%$ trabaja más de 40 horas semanales (Figura 5). 
Figura 5. Distribución de estudiantes con trayectorias de logro según hs. de trabajo semanales

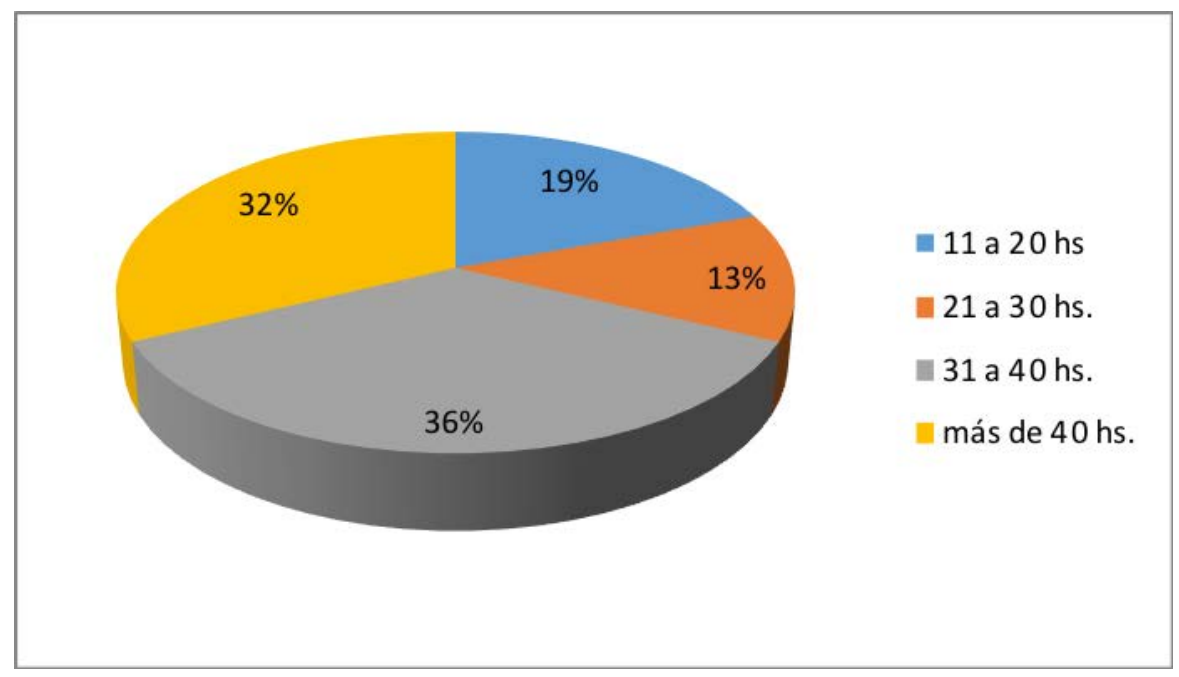

Dados estos resultados, tampoco podríamos decir que la disponibilidad de tiempo exclusivo para dedicar al estudio sea una variable altamente relacionada con el logro de trayectorias exitosas en la modalidad a distancia. De hecho, más de la mitad del grupo de los encuestados lograron compatibilizar actividades laborales demandantes en tiempo con trayectorias de logro en el cursado de sus estudios.

f. Costeo de los estudios. El grupo mayoritario (50\%) sostiene económicamente sus estudios con recursos provenientes de su propio trabajo. Otro $26 \%$ lo hace exclusivamente con aportes monetarios de su familia, en tanto que el porcentaje restante combina más de una fuente de recursos para el sostenimiento de sus estudios (por ejemplo, trabajo y becas, trabajo y planes sociales, etc.). Así, pareciera que no es necesaria una situación económica holgada para lograr trayectorias de éxito en la universidad; de hecho, dentro del grupo de estudiantes exitosos, algunos necesitan recurrir a becas, planes sociales o ayuda familiar para lograr el objetivo de mantenerse estudiando. Incluso otros indicadores del nivel socioeconómico del grupo sugieren que al menos algunos de los sujetos no provienen de contextos económicos favorecidos. Por ejemplo, en el $31 \%$ de los hogares de procedencia no se cuenta con ningún automóvil.

g. Aspectos motivacionales: las metas. Uno de los ítems del cuestionario administrado solicitaba a los estudiantes identificar sus metas como alumnos universitarios mediante la selección de uno de cinco relatos que representan la 
voz de hipotéticos estudiantes ${ }^{2}$. El siguiente gráfico presenta la distribución de las respuestas.

Figura 6. Distribución de estudiantes con trayectorias de logro según metas $(\mathrm{N}=46)$

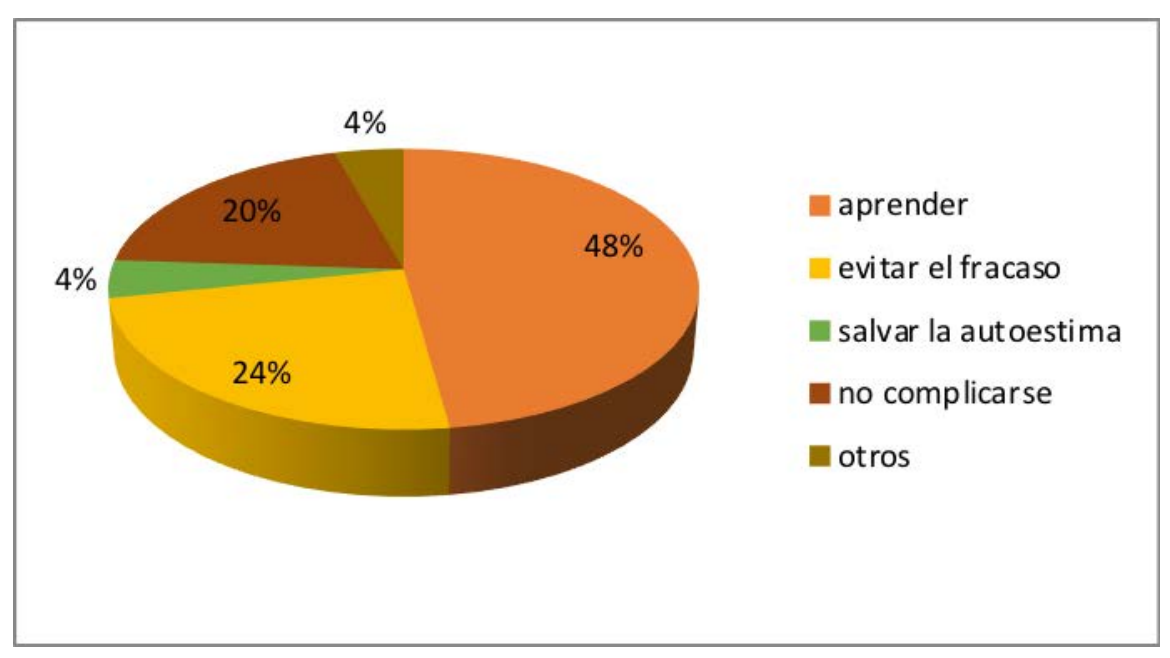

Como es esperable dentro de un grupo de estudiantes con buenos resultados académicos, la mayor parte de los sujetos $(48 \% ; \mathrm{N}=22)$ se orientó hacia el aprendizaje (figura 6). Las siguientes expresiones ilustran lo dicho: 'elegí la carrera porque siempre quise hacerla... es un objetivo importante en mi vida'; 'me interesa todo lo relacionado con lo económico, las estadísticas, los números. Es algo que disfruto'.

Si consideramos que en un estudio realizado con un grupo general de ingresantes (Chiecher, 2017) el porcentaje de quienes se orientaban hacia metas de aprendizaje fue del $32 \%$, podríamos pensar que estamos frente a una variable probablemente asociada con el éxito académico; pues en este grupo de estudiantes exitosos el porcentaje asciende a un $48 \%$.

h. Aspectos motivacionales: las atribuciones causales. Con el objeto de conocer sobre los procesos atribucionales realizados por los sujetos que obtuvieron buenos resultados académicos, el cuestionario administrado preguntaba a los estudiantes a qué atribuyen los buenos resultados logrados, sin sugerir alternativas de respuesta alguna; esto es, cada uno respondía libremente. Las respuestas, analizadas cualitativamente, fueron agrupadas en las siguientes categorías: 
- Atribución a causas internas y manejables por el sujeto. En esta categoría se ubican 35 sujetos (76\%) que refirieron a causas únicamente internas y/o personales para explicar los resultados académicos obtenidos. Esfuerzo, dedicación y tiempo dedicado al estudio fueron las causas mencionadas para explicar el logro de buenos resultados. A continuación, una expresión de un alumno para ilustrar esta categoría: 'atribuyo mis logros al esfuerzo; a leer todos los días un poco; a ser responsable e ir estudiando de acuerdo a los cronogramas y actividades'.

- Atribución a una combinatoria de causas internas y externas. Un grupo de 6 sujetos (13\%) hicieron mención de causas tanto internas como externas para explicar el rendimiento logrado. Aquí, además de las cuestiones personales, se mencionaron ayudas familiares, apoyo de docentes y relaciones con compañeros como factores que contribuyen a explicar los logros. Ejemplo: los buenos resultados se los debo a la ayuda de mi familia, de mis profesores que estuvieron acompañándome en el cursado y gracias a todas las técnicas de estudio que apliqué y al tiempo que le dediqué a las tres materias'.

- Atribución a causas externas. Por fin, 5 sujetos (9\%) explicaron sus resultados evocando únicamente causas externas y fuera de su propio control. Ejemplo: 'atribuyo mis logros a la ayuda y apoyo de mi familia y compañeros, al trabajo de los profesores'.

\section{Variables contextuales facilitadoras desde la visión de los estudiantes}

a. Docentes que favorecen el logro. Frente a la pregunta ¿́cómo describiría a un buen profesor?, la mayor parte del grupo (33 sujetos, 72\%) acordó en señalar la modalidad de enseñanza que implementan los docentes como una cuestión clave. En este sentido, se destaca de manera reiterada la importancia de recibir respuestas rápidas y devoluciones acerca de las actividades que se presentan. A continuación, una respuesta ilustrativa: 'en mi opinión un buen profesor es aquel que planifica el proceso de aprendizaje de la materia, explica y despeja dudas, realiza devoluciones de las actividades que los alumnos realizamos; en el caso de la modalidad a distancia todas las devoluciones son muy importantes porque nos guía en este proceso de aprendizaje... es bueno que contesten con rapidez'.

Como contraste, al consultar por las características de un profesor que no ayudan a aprender, aparecieron reiteradamente menciones a las demoras en responder pedidos de ayuda. Ejemplo: 'cuando el profesor no responde a las dudas o consultas del alumno en tiempo y forma, se produce inestabilidad del alumno y debe recurrir a otras fuentes para comprender, a veces de manera equivocada'.

También en 19 casos (41\%) se destacó como aspecto positivo de un docente la interacción que establece con los estudiantes, incluyéndose en esta categoría 
aspectos tales como el interés por su progreso, el acompañamiento, guía y apoyo; 13 menciones (28\%) estuvieron asociadas con características personales de un buen docente que trascienden lo educativo, tales como paciencia, puntualidad, respeto, etc.; finalmente, 9 (20\%) menciones destacaron como aspecto positivo de un docente la motivación y pasión por lo que hace, lo cual repercute en el incentivo, motivación y entusiasmo que genera en sus estudiantes.

b. Tareas que favorecen la motivación y el logro. Al consultar mediante una pregunta abierta qué clases o tareas agradan a los estudiantes, encontramos que, llamativamente, aunque han elegido la modalidad a distancia para cursar sus estudios, el grupo mayoritario (17 de los 46 estudiantes, 37\%) valora por sobre cualquier otra actividad, los encuentros presenciales o visitas a la universidad. Ejemplo: 'como soy alumno a distancia, lo que más me gustó fue el encuentro presencial, porque una explicación del profesor no se cambia por nada'. De hecho, aunque la carrera se dicta a distancia, se organizan eventualmente encuentros presenciales, siendo también en esta modalidad los exámenes de las asignaturas.

c. Ayudas y apoyos que favorecen el logro. Al consultar mediante una pregunta abierta por las ayudas con que el estudiante contó para haber logrado cursar con éxito, las respuestas refirieron mayormente a ayudas provenientes desde fuera de la institución (39\%); más precisamente de la familia - en calidad de sostén para el cuidado de los hijos o como motivadora- e incluso de los jefes o empleadores que otorgan permisos, por ejemplo, para ausentarse en fechas de exámenes. La que sigue es una respuesta ilustrativa: 'por mi parte lo que me ayudó fue el apoyo de mi familia, de que si desapruebo te incentivan a seguir, a no bajar los brazos. Primero y principal la ayuda de mis padres que me dan techo y comida, además cuidan de mi hijo mientras no estoy'.

Sin embargo, también fueron mencionados como ayudas el apoyo recibido de algunos docentes (24\%), la interacción con los pares (20\%) y algunas decisiones institucionales que contribuyeron al éxito (11\%). A continuación, un ejemplo ilustrativo de cada categoría.

- Ayuda de los docentes: 'los profesores te ayudan y tienen mucha consideración si no llegas con un trabajo, saben que muchas veces tenemos dificultades para llegar a una actividad y nos reciben los trabajos'.

- Ayuda de compañeros: 'me ayudó haber encontrado y entablado vínculos con mis compañeros de la modalidad distancia'.

- Ayudas institucionales: 'me ayudó que las materias estuvieran divididas en tiempos, al finalizar una comienza otra'. 


\section{DISCUSIÓN Y CONCLUSIONES}

Es ya sabido que el primer año en la universidad, en cualquier carrera y modalidad, resulta un periodo complejo de transitar. Tal es así que este momento del ingreso universitario ha sido caracterizado como una situación de extrañamiento, en la que el ingresante se siente extranjero en una cultura que le resulta nueva y extraña (Ortega, 2011).

Si pensamos específicamente en el ingreso en modalidad a distancia, se agregan características - del contexto y también de los ingresantes- que contribuyen a configurar esta complejidad a la que aludimos. El ingreso en una carrera con modalidad a distancia implica, al igual que en modalidad presencial, un gran cambio y un esfuerzo de adaptación al nuevo contexto. Probablemente el alumno estudie desde su misma casa, un lugar que le es conocido y familiar, pero el gran esfuerzo a desplegar está en conocer una manera de estudiar y de aprender que quizás no ha experimentado nunca y en lograr relacionarse con otros (docentes y compañeros) también de una manera diferente. Más aún, la fuerte demanda de autorregulación del aprendizaje que exige ser alumno en un entorno virtual y, sumado a ello, las competencias tecnológicas que es necesario desarrollar para moverse con soltura en el ambiente educativo mediado tecnológicamente, son también importantes factores a considerar cuando estamos frente a ingresantes en carreras dictadas con modalidad a distancia (Ficco, Chiecher y Bersía, 2013). Así pues, no resulta sencillo ser un estudiante virtual y menos aún lograr el ajuste de las trayectorias académicas reales a los tiempos teóricos pautados por el plan de estudios de las carreras. Son escasos los estudiantes que logran seguir el ritmo estipulado por el plan de estudios; según datos de este estudio tan solo un $9 \%$ del total de los inscriptos de tres cohortes logró regularizar las asignaturas previstas para el primer cuatrimestre. Las cifras hablan por sí mismas.

En el contexto descripto - que no es privativo de una carrera o institución en particular sino típico de la educación a distancia- se torna crucial intentar alguna respuesta a las preguntas: ¿cómo favorecer el logro en una mayor cantidad de estudiantes en esta modalidad de estudio? ¿Es posible intervenir institucionalmente? ¿Es posible intervenir desde la enseñanza?

En este artículo se ha descripto a un grupo de estudiantes exitosos, que consiguieron cursar trayectorias de logro, al menos en el primer tramo de sus carreras. Los resultados muestran que, entre las variables personales consideradas, aquellas que parecen tener vinculación con mejores rendimientos son el nivel educativo de los padres, las metas de aprendizaje y las atribuciones causales internas y modificables. No obstante, más allá de aquello que el estudiante puede aportar de sí mismo para el logro académico, también se identificaron características de los docentes y del contexto en general que podrían promover el logro.

Así, sobre algunas cuestiones, como por ejemplo el nivel educativo de los padres, no resulta posible incidir, pues ya vienen dadas de antemano y poco se puede 
hacer por cambiarlas. En cambio, sobre otras variables sí resultaría posible incidir institucionalmente procurando promover el logro académico de mayor cantidad de estudiantes. Con base en los resultados hallados en este estudio, algunas de las acciones que resultaría oportuno diseñar y llevar a la práctica tendrían que ver con:

- Capacitar a los docentes para el diseño de contextos pedagógicos de calidad, que fomenten la interacción, la motivación hacia el aprendizaje y la autorregulación. La educación a distancia ha sido históricamente "mirada de reojo", con recelo, desconfianza, al punto de cuestionarse incluso su calidad (siempre tomando como eje comparativo la educación presencial). Sin embargo, la calidad de la enseñanza no pasa por el contexto donde se desarrolle, sino por las características de la propuesta pedagógica que el docente instrumente. Así, es fundamental la formación pedagógica de los docentes para el diseño de propuestas de enseñanza que se ajusten a estándares de calidad. La carencia de formación pedagógica de muchos docentes universitarios resulta tal vez más aguda si pensamos en la enseñanza en entornos virtuales; esto es, si las carreras de grado no incluyen espacios de formación pedagógica, mucho menos consideran la formación para la enseñanza en un contexto específico como el virtual. Sin embargo, enseñar a distancia implica atender cuidadosamente a ciertas variables críticas, tales como la multiplicidad de roles que desempeña el tutor, las características a las que deberían ajustarse las e-actividades propuestas para promover la motivación y el aprendizaje, los estilos de interacción más convenientes, las herramientas que ofrecen las plataformas -y que el docente puede gestionar para el seguimiento y monitoreo del aprendizaje de los estudiantes-, y los modos de evaluación.

- Implementar un sistema de evaluación del perfil cognitivo, motivacional y de habilidades tecnológicas de los estudiantes antes de iniciar el cursado y ofrecer una devolución ofeedback individual a cada estudiante, marcando sus fortalezas y debilidades para estudiar en un contexto mediado tecnológicamente.

- Concientizar a los estudiantes, tal vez en talleres iniciales de sensibilización, acerca de la importancia del buen uso y de la necesidad de disponer de un tiempo diario para atender al estudio, procurando romper, de este modo, con el mito de que estudiar a distancia es más fácil o lleva menos tiempo.

- Implementar sistemas de evaluación del desempeño de los docentes en la formación virtual. En tal sentido, los estudiantes son informantes claves en su calidad de beneficiarios directos de la acción formativa. Recuperar sus voces, tal como lo hemos hecho en este estudio, aporta datos significativos para la mejora de la enseñanza. Asimismo, otra dimensión de la evaluación del desempeño docente debería estar dada por la autoevaluación que ellos mismos debieran hacer sobre sus prácticas. A título de ejemplo, en Salinas (2017) se describe un caso de creación e implementación de un sistema de evaluación de las prácticas de enseñanza desarrolladas en las aulas virtuales, en el cual se toman en cuenta la perspectiva de los alumnos y de los propios docentes. 
- Implementar, en la medida que sea posible, instancias presenciales complementarias, de carácter optativo, para aquellos estudiantes que deseen, necesiten y puedan concurrir. De hecho, los mismos estudiantes valoraron muy positivamente las instancias y encuentros presenciales complementarios a la virtualidad. De no ser posible, podrían ser sustituidas, por encuentros virtuales sincrónicos.

- Disponer de una figura de colaborador del profesor, o ayudante, que pueda complementarlo en la función del seguimiento individual de los estudiantes, tomando en cuenta los datos sobre la actividad de los usuarios que registran automáticamente las plataformas educativas, con el fin de optimizar los procesos de enseñanza y aprendizaje. La finalidad última es que la información obtenida guíe y oriente las actuaciones de los estudiantes, ayudándoles, por ejemplo, a regular su propia actividad o a coordinarse con el resto de miembros del grupo durante la realización de las actividades de aprendizaje (Coll, Engel y Niño, 2017); o bien, que los docentes la utilicen para orientar sus intervenciones, para tomar decisiones sobre cuándo sus estudiantes necesitan apoyos específicos o para detectar incomprensiones o patrones de actuación irregulares.

En síntesis, si bien gran parte del éxito en el aprendizaje en entornos virtuales tiene que ver con el estudiante, sus características, su motivación y su capacidad de dirigir y gestionar su propio proceso de aprendizaje, cabe reconocer que la calidad del diseño pedagógico parece también un factor decisivo si se quiere promover el logro de una mayor cantidad de estudiantes.

Como decíamos en la sección inicial del escrito, las variables que sostienen el logro estarían vinculadas con características personales de los estudiantes, pero también con variables contextuales o apoyos externos. Por ejemplo, la calidad de las tutorías y las interacciones profesor-alumno, la fuerte presencia docente o presencia didáctica, la implementación de ciertos estilos de interacción docente-alumno, el diseño de e-actividades capaces de promover motivación, entre otras. El accionar desde el contexto está entonces en manos de las instituciones, desde donde se pueden implementar numerosas acciones capaces de marcar la diferencia empoderando, sobre todo, a aquellos estudiantes que no llegan tal vez con todas las habilidades o la motivación necesarias para cursar estudios a distancia.

\section{NOTAS}

Contactar con la autora para acceder a la versión online del cuestionario referido.

2. Los relatos están disponibles en la versión completa del cuestionario. Recuperado de https://docs.google.com/forms/d/e/1FAIpQLScRcUjDi3dkaOT5bzWiV5L8fY KHsZHj2Z7DCBuqMmuWt-4Zw/viewform 


\section{REFERENCIAS}

Benett, C., y Monds, K. (2008). Online courses. The real challenge is 'motivation'. College Teaching Methods \& Styles Journal, 4(6), 1-6.

Carballo Aguilar, O. (2017). Factores que inciden en la permanencia académica de estudiantes a distancia en el nivel superior. Caso de estudio: Licenciatura en Estadística y Sistemas de Información. Revista Iberoamericana de Producción Académica y Gestión Educativa, 4(8), 1-21. Recuperado de http://www. pag.org.mx/index.php/PAG/article/ viewFile/694/924

Castejón, J. L., Gilar, R., Miñano, P., y Veas, A. (2016). Identificación y establecimiento de las características motivacionales y actitudinales de los estudiantes con rendimiento académico menor de lo esperado según su capacidad (underachievement). European Journal of Education and Psychology, 9(2), 47-88. Recuperado de https://bit.ly/2uZdJNE

Bossolasco, M. L., Chiecher, A. y Dos Santos, D. (en prensa). Análisis de trayectorias académicas en el primer año universitario. Validación de un cuestionario mediante juicio de expertos. Educación Superior, 27(1).

Coll Salvador, C., Engel Rocamora, A., y Niño Carrasco, S. (2017). La actividad de los participantes como fuente de información para promover la colaboración. Una analítica del aprendizaje basada en el modelo de influencia educativa distribuida. RED, Revista de Educación a Distancia, 53(2), 1-36. doi: http://dx.doi. org $/ 10.6018 / \mathrm{red} / 53 / 2$

Chiecher, A. (2017). Estudiantes de Ingeniería. Perfiles asociados con trayectorias de logro. En M. Panaia (Coord.), De la formación al empleo. Nuevos desafios e innovación (71-92). Buenos Aires: Miño y Dávila.
Chiecher, A., Ficco, C., Paoloni, P., y García, G. (2016). ¿Qué mueve a los estudiantes exitosos? Metas y motivaciones de universitarios en las modalidades presencial y distancia. Revista Observatorio, 2(1), 301-326. Recuperado de https://bit.ly/2UMVOIm

Chiecher, A., y Donolo, D. (2013). Trabajo grupal mediado por foros. Aportes para el análisis de la presencia social, cognitiva y didáctica en la comunicación asincrónica. En A. Chiecher, D. Donolo y J. L. Córica (Eds.), Entornos Virtuales y Aprendizaje. Nuevas perspectivas de estudio $e$ investigaciones (151-198). Mendoza: Editorial Virtual Argentina.

Chiecher, A., Paoloni, P., y Ficco, C. (2014). Ingreso a la universidad en modalidad a distancia. El papel de aspectos motivacionales y cognitivos en la configuración de logros académicos. Revista de Educación a Distancia, 43, 1-25. Recuperado de http://www.um.es/ ead/red/43/chiecher et al.pdf

Ficco, C., Chiecher, A., y Bersía, P. (2013). El ingreso en carreras con modalidad a distancia. Descripción de un dispositivo de seguimiento para identificar factores potencialmente desencadenantes del abandono. VI Seminario Internacional de Educación a Distancia. Recuperado de http://www.uncu.edu.ar/seminario rueda/upload/t15.pdf

Garbanzo Vargas, G. (2007). Factores asociados al rendimiento académico en estudiantes universitarios, una reflexión desde la calidad de la educación superior pública. Revista Educación, 31(1), 43-63. Recuperado de https://www.redalyc.org/ pdf/440/44031103.pdf

Garrison, R., Anderson, T., y Archer, W. (2000). Critical inquiry in a text-based environment: computer conferencing in higher education. The Internet and 
Higher Education, 2, 87-105. doi: https:// doi.org/10.1016/S1096-7516(00)00016-6

Garrison, R., Anderson, T., y Archer, W. (2003). A theory of critical inquiry in online distance education. En M. Moore y T. Anderson, (Eds.), Handbook of Distance Education (113-128). New Jersey: Lawrence Eilbaum Associates.

Huertas, J. A. (1997). Motivación. Querer aprender. Buenos Aires: Aique.

Huertas, J. A., y Agudo, R. (2003). Concepciones de estudiantes universitarios sobre la motivación. En C. Monereo y J. I. Pozo, (Coords.), La Universidad ante la nueva cultura educativa (45-62). Barcelona: Ed. Síntesis.

Kizilcec, R. F., Pérez-Sanagustín, M., y Maldonado, J. J. (2017). Self-regulated learning strategies predict learner behavior and goal attainment in Massive Open Online Courses. Computers \& Education, 104, 18-33. doi: https://doi. org/10.1016/j.compedu.2016.10.001

Onrubia, J. (2016). Aprender y enseñar en entornos virtuales: actividad conjunta, ayuda pedagógica y construcción del conocimiento. RED. Revista de Educación a Distancia, 5o(3), 1-14. doi: http:// dx.doi.org/10.6018/red/50/3

Ortega, F. (2011). Ingreso a la universidad. Relación con el conocimiento $y$ construcción de subjetividades. Córdoba: Ferreyra Editor.

Park, J., y Choi, H. (2009). Factors influencing adult learners' decision to drop out or persist in online learning. Educational Technology \& Society, 12(4), 207-217. Recuperado de https://www.jets.net/ETS/journals/12 4/18.pdf

Pintrich, P. (2000). The role of goal orientation in self-regulated learning. En M. Boekaerts, P. Pintrich y M. Zeidner, (Comps.), Handbook of Self-regulation. Theory, Research and Applications (451502). San Diego: Academic Press.

Rivera Montalvo, D. (2011). Factores que inciden en la retención o deserción del estudiante a distancia. Documento en Internet. Recuperado de http://ponce. inter.edu/cai/tesis/derivera/index.pdf

Romero, M., y Barberá, E. (2013). Identificación de las dificultades de regulación del tiempo de los estudiantes universitarios en formación a distancia. RED, Revista de Educación a Distancia, 38, 1-17. Recuperado de https://www. um.es/ead/red/38/romero barbera.pdf

Salinas, M. I. (2017). Gestión de la evaluación del desempeño docente en aulas virtuales de un proyecto de blended-learning. Ciencia, Docencia y Tecnología, 28(54) 100-129. Recuperado de https://www. redalyc.org/pdf/145/14551170004.pdf

Sepúlveda, P. (2016). Trayectorias reales en tiempos virtuales. Estudiantes y docentes universitarios desde una mirada inclusiva. Buenos Aires: Universidad Nacional de Quilmes. Recuperado de https://bit.ly/2uXtCE4

\section{PERFIL ACADÉMICO Y PROFESIONAL DE LA AUTORA}

Analía Claudia Chiecher Costa. Doctora en Psicología por la Universidad Nacional de San Luis; Magíster en Educación y Universidad y Licenciada en Psicopedagogía por la Universidad Nacional de Río Cuarto. Investigadora Independiente del Consejo Nacional de Investigaciones Científicas y Técnicas (CONICET, Argentina). Líneas de trabajo: aprendizaje en entornos virtuales, trayectorias académicas de estudiantes universitarios.

E-mail: achiecher@hotmail.com 
Dirección:

Juan B. Justo 925, Río Cuarto

Argentina, (5800)

Fecha de recepción del artículo: 07/01/2019

Fecha de aceptación del artículo: 25/01/2019

Fecha de aprobación para maquetación: 07/04/2019 